\section{Dosimetry of Critical Organs in Maxillofacial Imaging with Cone-beam Computed Tomography}

\author{
Ghanbarnezhad Farshi R. ${ }^{1,2}$, Mesbahi A. ${ }^{2}$, Johari M. ${ }^{3}$, Kara \\ Ü. ${ }^{4}$, Gharehaghaji N. ${ }^{5 *}$
}

\begin{abstract}
Background: While the benefits of cone-beam computed tomography (CBCT) are well known in maxillofacial imaging, the use of this modality is not risk-free.

Objective: The aim of this study was to evaluate the exposure doses received by patients during maxillofacial imaging with CBCT.

Methods: Entrance surface dose (ESD) was measured by using thermoluminescent dosimeters (TLDs) attached to the eyes lids, parotid glands and thyroid of 64 patients in two imaging centers (A and B). Phantom dosimetry was performed by a cylindrical poly-methyl methacrylate (PMMA) head-size phantom and an ionization chamber for different exposure parameters. NewTom VGi and Planmeca Promax 3D CBCT scanners were used at centers $\mathrm{A}$ and $\mathrm{B}$, respectively.
\end{abstract}

Results: The mean ESD of the eyes, parotid glands and thyroid were 2.57, 2.33 and $0.28 \mathrm{mGy}$ in center A, 0.35, 2.11 and $0.37 \mathrm{mGy}$ in center B, respectively. ESD of the eyes revealed a significant difference in two centers; in center B, it was $86.4 \%$ lower than center A. In the phantom dosimetry, the measured doses of NewTom VGi were 2.63 and $2.08 \mathrm{mGy}$, respectively by changing field of view (FOV) size from $8 \times 8 \mathrm{~cm}^{2}$ (height $\times$ diameter) to $6 \times 6 \mathrm{~cm}^{2}$. For Planmeca Promax $3 \mathrm{D}$, it ranged from 0.98 to $3.24 \mathrm{mGy}$ depending on exposure parameters.

Conclusion: There is a wide range of radiation doses dependent on the units, patients and selected scan parameters. Inappropriate selection of exposure settings, especially FOV size, can seriously increase patient dose.

\section{Keywords}

Cone-Beam Computed Tomography, Radiation Dosimetry, Entrance Surface Dose, Thermoluminescent Dosimetry, Maxillofacial Imaging, Dentistry

\section{Introduction}

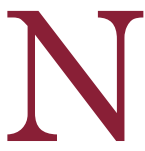
owadays, the use of X-ray as a reliable diagnostic tool in medical practice is inevitable [1-3]. Maxillofacial disease is one of the most common medical problems in communities which is detectable by the use of X-rays. Various radiography techniques are used for diagnosing and monitoring the treatment process in dental procedures. However, due to the complicated anatomy of maxillofacial region, a change from two-dimensional (2D) to three-dimensional (3D) imaging provides more anatomical details from treatment region $[4,5]$. More recently, a technology utilizing a cone-shaped X-ray beam and flat-panel detectors which is called cone-beam computed tomography
${ }^{1}$ Immunology Research

Center, Tabriz University

of Medical Sciences,

Tabriz, Iran

${ }^{2}$ Medical Physics Depart-

ment, Faculty of Medi-

cine, Tabriz University

of Medical Sciences,

Tabriz, Iran

${ }^{3}$ Department of Oral \&

Maxillofacial Radiology,

Faculty of Dentistry, Ta-

briz University of Medical

Sciences, Tabriz, Iran

${ }^{4}$ Vocational School of

Health Services, Suley-

man Demirel University,

Isparta, Turkey

${ }^{5}$ Radiology Department,

Paramedical Faculty, Ta-

briz University of Medical

Sciences, Tabriz, Iran

*Corresponding author:

N. Gharehaghaji,

Associate Professor

of Medical Physics,

Radiology Department,

Paramedical Faculty, Ta-

briz University of Medica

Sciences, Tabriz, Iran

E-mail: gharehaghaji@

gmail.com

Received: 23 November 2016 Accepted: 21 January 2017 
(CBCT) has been developed for 3D imaging in dental procedures [6]. In addition to CBCT diagnostic advantages to $2 \mathrm{D}$ imaging modalities, its smaller size, lower price and lower radiation dose to the patient compared to multidetector computed tomography (MDCT) scanners making it a desirable device for different clinical applications, including maxillofacial imaging centers [7-11].

During CBCT scans, patients are exposed to the ionizing radiation that is generally higher than conventional radiography [12]. In maxillofacial imaging, the parotid glands, eyes and thyroid gland as critical organs are inside or adjacent to the radiation area [13]. This issue has raised concerns about the biological risks of radiation dose with CBCT. To address this problem, in 2011, a dose measuring methodology for calculating the dose index (DI) for dental CBCT was suggested by "Safety and efficacy of a new and emerging dental X-ray modality (SEDENTEXCT)" project that could be measured and used to establish diagnostic reference level (DRL). It can be useful for the optimization of exposure settings to develop the radiation safety of patients who need CBCT imaging [14]. Moreover, several studies have been conducted on dosimetry using an anthropomorphic phantom, and effective dose has been reported [15-24]. Although this method is a useful approach to represent the overall risk from radiation exposure, it has some drawbacks, such as ignoring the influence of the demographical and anatomical factors of each patient on radiation-induced risks. Whereas patients will receive different doses regarding their sizes and masses as important factors, considering variation in patient dose is not easily carried out using a standard phantom which has a fixed size, mass and gender of a reference patient [25-27]. Especially for children whose tissue sizes and positions are different from adults and in similar exposure settings, the absorbed dose to different organs in the head and neck regions may be higher than adults, because more tissues will be exposed to the primary beam $[25,28]$. Another shortcoming of phantom studies is that they may not completely show the clinical reality and variability of CBCT radiation dose which patients receive in actual clinical settings. Particularly in situations in which imaging centers do not use specific guidelines for CBCT exposures, radio-technologists do not use appropriate exposure settings for different diagnostic purposes. Although these factors can extremely increase the patient's dose, the role of them is not usually considered in studies done on the phantom.

Measurement of patients' dose in X-ray examinations especially radiographic procedures have been widely done in different imaging centers to determine real radiation dose which received by the patients [29-32]. But there are few studies based on dose measurement on patients undergoing CBCT imaging [25].

The aim of this study was to obtain entrance surface dose (ESD) in sensitive organs of head and neck region on patients undergoing CBCT examinations at two main maxillofacial imaging centers in East Azerbaijan for the first time. Evaluation of the appropriate exposure parameters for patient's dose reduction was also conducted using dose measuring methodology (SEDENTEXCT project). We hope this study promotes to perform similar countrywide studies for the evaluation of a national diagnostic reference level (NDRL) for maxillofacial CBCT imaging in Iran.

\section{Material and Methods}

\section{CBCT Scanners}

Two CBCT units were included in this study; exposure parameters varied between patients and between CBCT models; the first group of scans was performed by NewTom VGi (Quantitative Radiology, Verona, Italy) in center A. In this unit, field of view (FOV) could vary from the smallest size of $6 \times 6 \mathrm{~cm}^{2}$ 
(height $\times$ diameter) to the largest of $15 \times 15$ $\mathrm{cm}^{2}$, and it could be selected directly from the software. Tube voltage was fixed at $110 \mathrm{kVp}$ and the mAs was determined through a type of automatic exposure control based on a 2D scout image. The second group of scans was performed by Planmeca Promax 3D CBCT (Planmeca OY, Helsinki, Finland) in center B. This $\mathrm{X}$-ray machine uses a tube voltage varying from 54 to 84 ( $2 \mathrm{kV}$ intervals), and $\mathrm{mA}$ from 8 to 16 (in 2-mA increments). FOV size could vary from $5 \times 4 \mathrm{~cm}^{2}$ to $8 \times 8 \mathrm{~cm}^{2}$.

\section{Patients}

In total, 64 patients were included involving two main maxillofacial imaging centers in East Azerbaijan. Two patient groups were studied: the first group consisted of 32 patients who referred to Oral and Maxillofacial Radiology, Faculty of Dentistry, Tabriz University of Medical Sciences (center A), and the second group consisted of 32 patients who referred to a private Oral and Maxillofacial Radiology Clinic in Tabriz (center B). All patients imaging was performed as routine examinations, patients were not exposed to any additional radiation, and the images were used for diagnostic purposes. There were no exclusion criteria regarding age, weight or other parameters. All patients are given an informed consent prior to the examination. The Ethical Committee of Tabriz University of Medical Sciences approved the ethical conditions of this study.

Before examination for all patients, demographical and anatomical data were recorded including gender, age, weight, height and chin-thyroid distance. The body mass index (BMI) for each patient was calculated using the equation: $\mathrm{BMI}=\frac{\text { Weight }(\mathrm{kg})}{(\text { Height }(\mathrm{m}))^{2}}$. Patients' information is summarized in Table 1. According to BMI, the patients were divided into four groups: Less than 18.5 as underweight, 18.5 to 25 as normal, 25 to 30 as overweight and above 30 are considered obese.

\section{Patient Dosimetry}

ESD was measured using $\mathrm{LiF}, \mathrm{Mg}, \mathrm{Cu}, \mathrm{P}$ TLD GR200 (Hangzhou, Zhejiang, China). Each chip was placed inside a plastic cover and specified with a special number. The sachets were attached to the seven points on the head and neck of each patient, including left and right eye lids, left and right parotid glands and on the three points of thyroid gland. In each examination, two additional TLDs were

Table 1: Demographical and anatomical information of patients

\begin{tabular}{|c|c|c|c|c|c|c|}
\hline & \multicolumn{3}{|c|}{ Center A } & \multicolumn{3}{|c|}{ Center B } \\
\hline & Male & Female & Total & Male & Female & Total \\
\hline Number of patients & 16 & 16 & 32 & 20 & 12 & 32 \\
\hline \multirow[t]{3}{*}{ Percentage $(\%)$} & 50 & 50 & 100 & 62.5 & 37.5 & 100 \\
\hline & \multicolumn{3}{|c|}{ Mean } & \multicolumn{3}{|c|}{ Mean } \\
\hline & Male & Female & Total & Male & Female & Total \\
\hline Age (years) & 45.25 & 52.5 & 48.8 & 48.4 & 51 & 49.14 \\
\hline Weight (kg) & 87.75 & 62.75 & 75.25 & 79.4 & 74.5 & 78 \\
\hline Height (cm) & 179.5 & 157.5 & 168.6 & 169.4 & 164.5 & 168 \\
\hline $\mathrm{BMI}\left(\mathrm{kg} \cdot \mathrm{m}^{-2}\right)$ & 27.26 & 25.19 & 26.22 & 27.83 & 27.55 & 27.75 \\
\hline Chin-thyroid distance $(\mathrm{cm})$ & 5.7 & 6.1 & 5.9 & 5.9 & 6.75 & 6.1 \\
\hline
\end{tabular}


used to estimate background radiation, which was subtracted from the measured dose values.

All processes related to TLDs (i.e. calibration, annealing and reading) were performed by Secondary Standard Dosimetry Laboratory (SSDL) of Karaj-Iran", and finally amount of dose was reported in mGy.

\section{Exposure Parameters in each Center}

In center $A$, tube voltage was fixed at 110 $\mathrm{kVp}$, and FOV was fixed at $15 \times 15 \mathrm{~cm}^{2}$, and for center $\mathrm{B}$ different tube voltages $(72-78 \mathrm{kVp})$ and mAs (146-160) were used, but the FOV was fixed at $8 \times 8 \mathrm{~cm}^{2}$.

\section{Phantom Dosimetry}

A cylindrical head-size phantom (UnforsRaySafe, Gothenburg, Sweden) with 16 $\mathrm{cm}$ diameter and $14 \mathrm{~cm}$ height and a penciltype ionization chamber (UnforsRaySafe, Gothenburg, Sweden) with an active length of 15 $\mathrm{cm}$ was used for this study. The phantom was made up of polymethyl methacrylate (PMMA). It contained five cylindrical slots for the inser- tion of an ionization chamber. Four homogeneous PMMA rods were provided for filling empty slots (Figure 1 (a)). For identification, each slot was assigned a code $(\mathrm{C}, \mathrm{P} 1, \mathrm{P} 2, \mathrm{P} 3$ and $\mathrm{P} 4)$; where $\mathrm{C}$ is the central hole and $\mathrm{P} 1$, P2, P3 and P4 are four peripheral holes (Figure 1 (b)). In each scan, the dose at each point was measured and dose wasobtained using the equation suggested by SEDENTEXCT project (Dose $\left.=\frac{\mathrm{C}+\mathrm{P}}{2}\right)$ where $\mathrm{P}$ is the average dose of peripheral hole $\left(\mathrm{P}=\frac{\mathrm{P} 1+\mathrm{P} 2+\mathrm{P} 3+\mathrm{P} 4}{4}\right)$. The isocenter of units must be at the $\mathrm{C}$ point (the center of the phantom).

\section{Statistical Analysis}

The independent t-test was used for values which had normal distribution, and MannWhitney $U$ and Kruskal-Wallis tests were implemented for values which were not normally distributed. For all tests, the level of significance $(\mathrm{P})$ was considered $<0.05$. Statistical
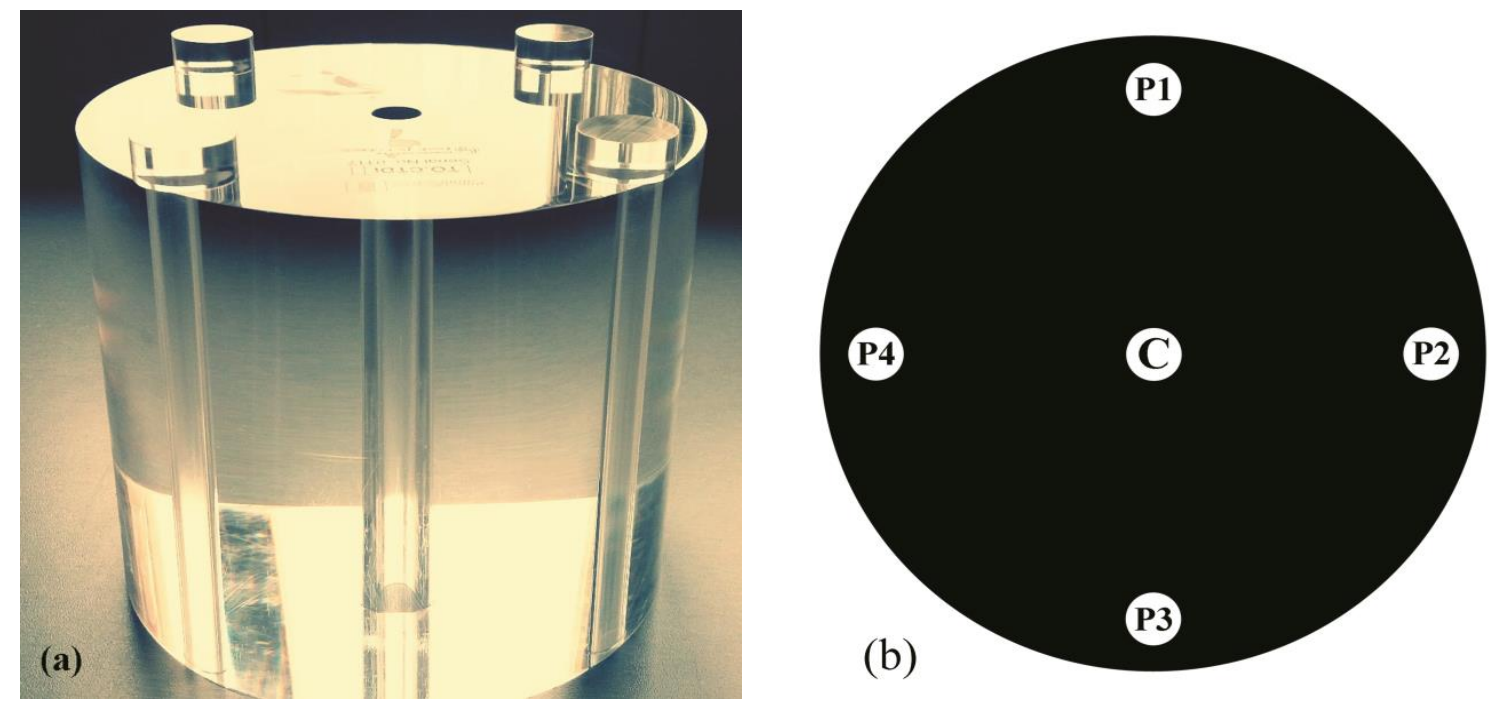

Figure 1: Phantom of cone-beam computed tomography ( $C B C T$ ) dosimetry. (a): A cylindrical head-size poly-methyl methacrylate (PMMA) phantom with five cylindrical slots for the insertion of an ionization chamber. (b): A schematic illustration of slot arrangement. 
analysis was performed with SPSS 16.

\section{Results}

\section{Patient's Dosimetry}

Table 2 summarizes results of ESD measurements for two CBCT units used in two centers in this study. Measured ESDs to symmetrical organs (eyes and parotid glands) were averaged.

In center $\mathrm{A}$, the highest and lowest doses were received by eyes and the thyroid gland, respectively, while in center B the highest and lowest doses were detected in the parotid glands and the eyes, respectively. A statistically significant difference was found between eye doses in two centers $(\mathrm{P}<0.001)$, the eye doses in center B were $86.4 \%$ lower than that of center A. Statistically significant differences were not found between the parotid glands and also thyroid gland doses in two centers $(\mathrm{P}$ $=0.35$ for parotid glands and $\mathrm{P}=0.18$ for thyroid gland).

For none of CBCT devices, between the BMI groups and doses, no statistically significant difference was detected $(\mathrm{P}>0.05)$. There was not any clear correlation between demographical and anatomical parameters and doses.

\section{Phantom Dosimetry}

A total of 8 exposure protocols were performed as summarized in Table 3. The ex-

Table 2: Entrance surface doses (mGy) for the eyes, parotid and thyroid glands for two maxillofacial imaging centers

\section{Center A}

\section{Center B}

\begin{tabular}{lcccccccc} 
& Min & Max & Mean & SD $^{*}$ & Min & Max & Mean & SD \\
\hline Eyes & 2.22 & 2.88 & 2.57 & 0.25 & 0.18 & 0.50 & 0.35 & 0.12 \\
\hline Parotid & 1.97 & 2.87 & 2.33 & 0.33 & 1.38 & 2.90 & 2.11 & 0.55 \\
\hline Thyroid & 0.22 & 0.38 & 0.28 & 0.06 & 0.18 & 0.62 & 0.37 & 0.16
\end{tabular}

*SD: Standard deviation

Table 3: Exposure parameters and doses for two $\mathrm{CBCT}$ devices in phantom dosimetry

Voltage (kV) Amperage (mA) Exposure time(s) FOV $\left(\mathrm{cm}^{2}\right)^{*}$ dose (mGy)

\begin{tabular}{lccccc}
\hline CBCT unit & 110 & Auto & Auto & $8 \times 8$ & 2.63 \\
\hline NewTom VGi & 110 & Auto & Auto & $6 \times 6$ & 2.08 \\
\hline Planmeca Promax 3D & 84 & 8 & 12 & $8 \times 8$ & 1.37 \\
\hline & 84 & 12 & 12 & $8 \times 8$ & 2.36 \\
\hline & 84 & 16 & 12 & $8 \times 8$ & 3.24 \\
\hline & 84 & 8 & 12 & $5 \times 8$ & 0.98 \\
\hline & 84 & 12 & 12 & $5 \times 8$ & 1.56 \\
\hline & 84 & 16 & 12 & $5 \times 8$ & 2.33
\end{tabular}

*FOV: field of view (height $\times$ diameter) 
aminations were repeated three times for each protocol to ensure the reproducibility of the measurements. Average of the three measurements was considered as the dose at each slot.

According to the Table 3, for NewTom VGi, $20.9 \%$ reduction was observed in the dose, by changing the FOV from $8 \times 8 \mathrm{~cm}^{2}$ to $6 \times 6$ $\mathrm{cm}^{2}$. For Planmeca Promax 3D, by changing $\mathrm{mA}$ from 8 to 12,12 to 16 and 8 to 16 with applying a fixed FOV of $8 \times 8 \mathrm{~cm}^{2}$, the amount of dose increased $42 \%, 37.3 \%$ and $57.7 \%$, respectively and when the FOV was $5 \times 8 \mathrm{~cm}^{2}$ with changing $\mathrm{mA}$ as mentioned, the dose increased $37.2 \%, 33 \%$ and $55.4 \%$, respectively. When $\mathrm{mA}$ was kept fixed at 8, 12 and 16, and while the FOV changed from $8 \times 8 \mathrm{~cm}^{2}$ to $5 \times 8$ $\mathrm{cm}^{2}$, dose reduction was $28.5 \%, 33.9 \%$ and $28.1 \%$, respectively.

\section{Discussion}

\section{Patient's Dosimetry}

As seen in Table 2 at center B, the maximum ESD belonged to parotid glands. As expected in CBCT imaging, parotid glands due to their locations were exposed to primary beam and received greater dose in comparison with two other organs which were out of radiation field and mainly received scattered radiation. However, the interesting point to note is that in center $\mathrm{A}$, eyes also received a high dose because of the large fixed FOV $\left(15 \times 15 \mathrm{~cm}^{2}\right)$ used in this center leading to exposure of the eyes with primary radiation beam. Despite the fact that the height and diameter of the scan field can have different values and there are a variety of choices for selecting the FOV of the CBCT devices, both centers in this study used a fixed FOV for all patients that led to an increase in the patients' dose. In a study performed by Pauwels et al. [25] measuring ESD in three imaging centers, the maximum doses for the eyes, parotid and thyroid glands were reported 2337, 2770, $2559 \mu \mathrm{Gy}$, and the minimum dose for these organs were 20,71,
$6 \mu \mathrm{Gy}$, respectively. The difference between measured ESDs in the present study and their study might be relevant to the type of CBCT units, exposure conditions, type, number and positioning of dosimeter and anatomical differences of patients. Based on the results, although the measured ESD for different patients was different, there was no clear correlation between demographical and anatomical factors and the measured dose. It seems that the effect of these factors on the dose is limited or overshadowed by other factors. Therefore, it can be concluded that the dose is a metric influenced by several factors such as different exposure parameters (FOV size and $\mathrm{mA}$ ) which can be dominant in comparison with the effect of demographical and anatomical factors on the dose.

Based on the hypothesis of radiation protection, any value of radiation dose can cause biological damage [13]. Therefore, CBCT device settings must be based on as low as reasonably achievable (ALARA) principles, which suggest strategies to dose reduction [16]. According to the International Commission on Radiological Protection (ICRP) guidelines, 2000 mGy may create temporary erythema; this dose is considered as the threshold for deterministic effects [33]. The maximum skin dose recorded in this study did not exceed 2.9 $\mathrm{mGy}$ that is about a 690 times smaller than the threshold. Although the allowed doses for the eyes and skin are similar but recent studies have shown that the risk of cataract increases with increasing dose, and threshold for cataract is less than $500 \mathrm{mGy}$ [33]. However, in the present study, the maximum dose to the eyes was $2.88 \mathrm{mGy}$ which is 174 times less than the suggested threshold.

Because in this study the thyroid was not exposed to the primary beam in both centers, the dose range was 0.18 to $0.62 \mathrm{mGy}$ which can be assumed as a low dose. Since thyroid is one of the critical organs and radiation can increase the risk of thyroid cancer [32], its 
dose should be reduced as much as possible. In the present study, in none of the centers any special protection for the thyroid and eyes was not used, while one of the suitable methods for dose reduction is the use of shielding (thyroid collars and leaded glasses). Goren et al. [34] found that by using thyroid shield and leaded glasses, thyroid and eye lens dose reduced $42 \%$ and $61 \%$, respectively.

\section{Phantom Dosimetry}

In this study for measurement and reporting the result of phantom dosimetry, we used the method suggested by the SEDENTEXCT project, because of its convenient execution that can be used easily for further studies to establish DRL.

Results of the present study demonstrated that by reducing the FOV from $8 \times 8 \mathrm{~cm}^{2}$ to $6 \times 6 \mathrm{~cm}^{2}$ in center $\mathrm{A}$, the dose reduced $20.9 \%$, and in center $\mathrm{B}$ by changing FOV from $8 \times 8$ $\mathrm{cm}^{2}$ to $5 \times 8 \mathrm{~cm}^{2}$, dose reduction was $28.5 \%$, $33.9 \%$ and $28.1 \%$ for the situation in which $\mathrm{mA}$ was fixed at 8,12 and 16, respectively. According to the results, it can be concluded that decreasing the FOV is one of the effective ways to reduce the dose. Previous studies on phantoms have shown that effective dose can be reduced by decreasing the FOV. In a study performed with Planmeca Promax device, in situations where only the FOV was changed and other exposure parameters were fixed, the results showed that if the height was limited to the mandible or maxilla, the effective dose reduced to $57 \%$ of the full height for mandible and $44 \%$ for maxilla. If the diameter was restricted just to the posterior or anterior areas, the effective doses would reduce to $66 \%$ and $42 \%$ of the full diameter [35].

In the present study for Planmeca Promax 3D (center B) while only the $\mathrm{mA}$ was changed and other parameters were fixed, when the FOVs were $8 \times 8 \mathrm{~cm}^{2}$ and $5 \times 8 \mathrm{~cm}^{2}$, the observed dose reduction was $57.7 \%$ and $55.4 \%$, respectively by decreasing $\mathrm{mA}$ from 16 to 8 . Therefore, one of the effective options for dose reduction is reducing the $\mathrm{mA}$. In principle, concerning the devices such as Planmeca Promax 3D in which $\mathrm{mA}$ can be selected manually by operators, the mA should be reduced as much as possible. Of course, reducing the mA must be in such a way that the image quality is also acceptable. In a study, Qu et al. [35] observed $65.8 \%$ reduction in effective dose by changing $\mathrm{mA}$ from 16 to 8 by use of Planmeca Promax device.

\section{Conclusion}

As evidenced in this study, a combination of scan parameters and patient's factors could have effects on the measured ESD, especially exposure parameters like FOV and $\mathrm{mA}$ settings which could change dose values; inappropriate selection of them can seriously increase patient's dose. Therefore, it is recommended to reduce the dose; the FOV should be selected as small as possible so that only the ROI is exposed to the primary beam, and other organs like eyes and thyroid gland have to be placed away from the primary beam. Another way for reducing the patient's dose is decreasing the $\mathrm{mA}$, but it should be in a condition that the image quality could be acceptable. The relationship between the $\mathrm{mA}$ and image quality of CBCT devices can be investigated in future studies. For dose reduction, development of guidelines and radiation protection principles and properly training of radio-technologists for the appropriate use of CBCT imaging based on the patient's needs, clinical issues and ALARA principles are indispensable. We hope the results of this study, with the help of similar future studies, lead to set NDRL for CBCT examinations in Iran.

\section{Acknowledgment}

This study was supported by a research fund from Immunology Research Center, Tabriz 
University of Medical Sciences. The authors are grateful to all staff of the centers and patients for their assistance and corporation.

\section{Conflict of Interest}

None

\section{References}

1. Heydarheydari S, Haghparast A, Eivazi MT. A Novel Biological Dosimetry Method for Monitoring Occupational Radiation Exposure in Diagnostic and Therapeutic Wards: From Radiation Dosimetry to Biological Effects. J Biomed Phys Eng. 2016;6:21-6. PubMed PMID: 27026951. PubMed PMCID: 4795325.

2. Khoshdel-Navi D, Shabestani-Monfared A, Deevband MR, Abdi R, Nabahati M. Local-Reference Patient Dose Evaluation in Conventional Radiography Examinations in Mazandaran, Iran. J Biomed Phys Eng. 2016;6:61-70. PubMed PMID: 27672626. PubMed PMCID: 5022756.

3. Janbabanezhad Toori A, Shabestani-Monfared A, Deevband MR, Abdi R, Nabahati M. Dose Assessment in Computed Tomography Examination and Establishment of Local Diagnostic Reference Levels in Mazandaran, Iran. J Biomed Phys Eng. 2015;5:177-84. PubMed PMID: 26688796. PubMed PMCID: 4681462.

4. Kiljunen $T$, Kaasalainen $T$, Suomalainen $A$, Kortesniemi M. Dental cone beam CT: A review. Phys Med. 2015;31:844-60. doi.org/10.1016/j. ejmp.2015.09.004. PubMed PMID: 26481816.

5. Nemtoi A, Czink C, Haba D, Gahleitner A. Cone beam CT: a current overview of devices. Dentomaxillofac Radiol. 2013;42:20120443. doi. org/10.1259/dmfr.20120443. PubMed PMID: 23818529. PubMed PMCID: 3922261.

6. Lavanya R, Babu DB, Waghray S, Chaitanya NC, Mamatha B, Nithika M. A Questionnaire Cross-Sectional Study on Application of CBCT in Dental Postgraduate Students. Pol J Radiol. 2016;81:181-9. doi.org/10.12659/PJR.895688. PubMed PMID: 27158283. PubMed PMCID: 4846182.

7. Yang CC. Characterization of Scattered X-Ray Photons in Dental Cone-Beam Computed Tomography. PLOS One. 2016;11:e0149904. doi. org/10.1371/journal.pone.0149904. PubMed PMID: 26950435. PubMed PMCID: 4780712.

8. Shahidi S, Bahrampour E, Soltanimehr E, Zamani A, Oshagh M, Moattari M, et al. The accuracy of a designed software for automated localization of craniofacial landmarks on CBCT images. BMC Med Imaging. 2014;14:32.

9. Rehani MM. Radiological protection in computed tomography and cone beam computed tomography. Ann ICRP. 2015;44:229-35. doi. org/10.1177/0146645315575872. PubMed PMID: 25816279.

10. Nardi C, Borri $C$, Regini $F$, Calistri L, Castellani $A$, Lorini $\mathrm{C}$, et al. Metal and motion artifacts by cone beam computed tomography (CBCT) in dental and maxillofacial study. Radiol Med. 2015;120:618-26. doi.org/10.1007/s11547-0150496-2. PubMed PMID: 25634792.

11. Jaju PP, Jaju SP. Cone-beam computed tomography: Time to move from ALARA to ALADA. Imaging Sci Dent. 2015;45:263-5. doi.org/10.5624/ isd.2015.45.4.263. PubMed PMID: 26730375. PubMed PMCID: 4697012.

12. Lee C, Lee SS, Kim JE, Symkhampha K, Lee WJ, Huh $\mathrm{KH}$, et al. A dose monitoring system for dental radiography. Imaging Sci Dent. 2016;46:1038. doi.org/10.5624/isd.2016.46.2.103. PubMed PMID: 27358817. PubMed PMCID: 4925646.

13. Cordasco G, Portelli M, Militi A, Nucera R, Lo Giudice A, Gatto E, et al. Low-dose protocol of the spiral CT in orthodontics: comparative evaluation of entrance skin dose with traditional Xray techniques. Prog Orthod. 2013;14:24. doi. org/10.1186/2196-1042-14-24. PubMed PMID: 24325970. PubMed PMCID: 4384968.

14. Araki K, Patil S, Endo A, Okano T. Dose indices in dental cone beam CT and correlation with dose-area product. Dentomaxillofac Radiol. 2013;42:20120362. doi.org/10.1259/ dmfr.20120362. PubMed PMID: 23520392. PubMed PMCID: 3635780.

15. Signorelli L, Patcas R, Peltomaki T, Schatzle M. Radiation dose of cone-beam computed tomography compared to conventional radiographs in orthodontics. J Orofac Orthop. 2016;77:9-15. doi.org/10.1007/s00056-015-0002-4. PubMed PMID: 26747662.

16. Nikneshan S, Aghamiri MR, Moudi E, Bahemmat N, Hadian H. Dosimetry of Three Cone Beam Computerized Tomography Scanners at Different Fields of View in Terms of Various Head and Neck Organs. Iran J Radiol. 2016;13:e34220. doi. org/10.5812/iranjradiol.34220. PubMed PMID: 27853498. PubMed PMCID: 5107245.

17. Soares MR, Batista WO, Antonio Pde L, Caldas 
$L V$, Maia AF. Study of effective dose of various protocols in equipment cone beam CT. Appl Radiat Isot. 2015;100:21-6. doi.org/10.1016/j.apradiso.2015.01.012. PubMed PMID: 25665897.

18. Kadesjo N, Benchimol D, Falahat B, Nasstrom K, Shi $X Q$. Evaluation of the effective dose of cone beam CT and multislice CT for temporomandibular joint examinations at optimized exposure levels. Dentomaxillofac Radiol. 2015;44:20150041. doi.org/10.1259/dmfr.20150041. PubMed PMID: 26119344. PubMed PMCID: 4628419.

19. Chambers D, Bohay R, Kaci L, Barnett R, Battista J. The effective dose of different scanning protocols using the Sirona GALILEOS $((\mathrm{R}))$ comfort CBCT scanner. Dentomaxillofac Radiol. 2015;44:20140287. doi.org/10.1259/ dmfr.20140287. PubMed PMID: 25358865. PubMed PMCID: 4614170.

20. Batista WO, Soares MR, de Oliveira MV, Maia AF, Caldas LV. Assessment of protocols in cone-beam CT with symmetric and asymmetric beams usingeffective dose and air kerma-area product. Appl Radiat Isot. 2015;100:16-20. doi. org/10.1016/j.apradiso.2015.01.014. PubMed PMID: 25620114.

21. Pauwels R, Zhang G, Theodorakou C, Walker A, Bosmans $H$, Jacobs $R$, et al. Effective radiation dose and eye lens dose in dental cone beam CT: effect of field of view and angle of rotation. $\mathrm{Br}$ J Radiol. 2014;87:20130654. doi.org/10.1259/ bjr.20130654. PubMed PMID: 25189417. PubMed PMCID: 4170857.

22. Hofmann E, Schmid M, Sedlmair M, Banckwitz R, Hirschfelder U, Lell M. Comparative study of image quality and radiation dose of cone beam and low-dose multislice computed tomography-an in-vitro investigation. Clin Oral Investig. 2014;18:301-11. doi.org/10.1007/s00784-0130948-9. PubMed PMID: 23460022.

23. Rottke D, Patzelt S, Poxleitner P, Schulze D. Effective dose span of ten different cone beam CT devices. Dentomaxillofac Radiol. 2013;42:20120417. doi.org/10.1259/dmfr.20120417. PubMed PMID: 23584925. PubMed PMCID: 3699973.

24. Pauwels R, Beinsberger J, Collaert B, Theodorakou C, Rogers J, Walker A, et al. Effective dose range for dental cone beam computed tomography scanners. Eur J Radiol. 2012;81:267-71. doi. org/10.1016/j.ejrad.2010.11.028. PubMed PMID: 21196094.

25. Pauwels $R$, Cockmartin $L$, Ivanauskaite $D$, Urboniene A, Gavala S, Donta C, et al. Estimat- ing cancer risk from dental cone-beam CT exposures based on skin dosimetry. Phys Med Biol. 2014;59:3877-91. doi.org/10.1088/00319155/59/14/3877. PubMed PMID: 24957710.

26. Cassola VF, Milian FM, Kramer R, de Oliveira Lira $\mathrm{CA}$, Khoury HJ. Standing adult human phantoms based on 10th, 50th and 90th mass and height percentiles of male and female Caucasian populations. Phys Med Biol. 2011;56:3749-72. doi. org/10.1088/0031-9155/56/13/002. PubMed PMID: 21628776.

27. Marine PM, Stabin MG, Fernald MJ, Brill AB. Changes in radiation dose with variations in human anatomy: larger and smaller normalstature adults. J Nucl Med. 2010;51:806-11. doi.org/10.2967/jnumed.109.073007. PubMed PMID: 20395339. PubMed PMCID: 2931266.

28. Al Najjar A, Colosi D, Dauer LT, Prins R, Patchell G, Branets I, et al. Comparison of adult and child radiation equivalent doses from 2 dental cone-beam computed tomography units. $A m \mathrm{~J}$ Orthod Dentofacial Orthop. 2013;143:784-92. doi.org/10.1016/j.ajodo.2013.01.013. PubMed PMID: 23726328.

29. Sina S, Zeinali B, Karimipoorfard M, Lotfalizadeh F, Sadeghi M, Zamani E, et al. Investigation of the entrance surface dose and dose to different organs in lumbar spine imaging. J Biomed Phys Eng. 2014;4:119-26. PubMed PMID: 25599058. PubMed PMCID: 4289519.

30. Mortazavi SMJ, Shareghi A, Kavousi A, GhiassiNejad M, Jafari-Zadeh M, Nazeri F, et al. The need for national diagnostic reference levels: Entrance surface dose measurement in intraoral radiography. Iran J Radiat Res. 2004;2:127-33.

31. Zenobio EG, Zenobio MA, Nogueira MS, Silva TA, Shibli JA. Absorbed radiation doses during tomographic examinations in dental implant planning: a study in humans. Clin Implant Dent Relat Res. 2012;14:366-72. doi.org/10.1111/j.17088208.2010.00277.x. PubMed PMID: 20491821.

32. Bahreyni Toossi MT, Akbari F, Bayani Roodi S. Radiation exposure to critical organs in panoramic dental examination. Acta Med Iran. 2012;50:80913. PubMed PMID: 23456522.

33. Akyalcin S, English JD, Abramovitch KM, Rong XJ. Measurement of skin dose from cone-beam computed tomography imaging. Head Face Med. 2013;9:28. doi.org/10.1186/1746-160X-9-28. PubMed PMID: 24192155. PubMed PMCID: 3851731. 
34. Goren AD, Prins RD, Dauer LT, Quinn B, Al-Najjar A, Faber RD, et al. Effect of leaded glasses and thyroid shielding on cone beam CT radiation dose in an adult female phantom. Dentomaxillofac Radiol. 2013;42:20120260. doi.org/10.1259/ dmfr.20120260. PubMed PMID: 23412460. PubMed PMCID: 3667524.
35. Qu XM, Li G, Ludlow JB, Zhang ZY, Ma XC. Effective radiation dose of ProMax 3D cone-beam computerized tomography scanner with different dental protocols. Oral Surg Oral Med Oral Pathol Oral Radiol Endod. 2010;110:770-6. doi. org/10.1016/j.tripleo.2010.06.013. PubMed PMID: 20952220. 